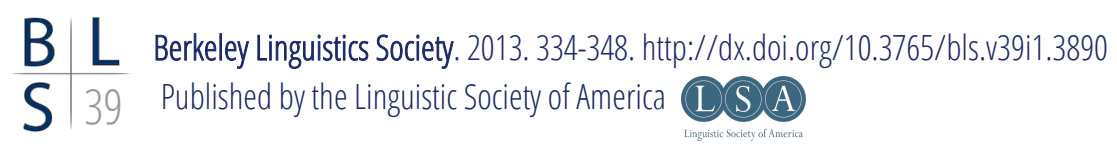

\title{
Anchored to what? An anaphoric approach to frames of reference
}

\author{
Jefferson Barlew* \\ The Ohio State University
}

\section{Introduction}

Spatial expressions are often used to specify the location of an entity. Following Talmy (1985), that entity is called the Figure. In (1) the location of the ball is being specified, and therefore the ball is the Figure.

(1) The ball is behind the car.

Many spatial expressions, including behind in (1), specify the location of the Figure in terms of its relation to the location of another entity, known as the Ground (Talmy 1985). In (1), the location of the Figure (the ball) is specified in terms of its relation to the location of the car. Thus, the car is the Ground.

Spatial expressions can be classified as non-projective or projective (Herskovits 1986). Non-projective expressions, such as English in and near, encode nondirectional spatial relations between the Figure and the Ground (see Kracht 2002

*I want to express a heartfelt thanks to the people who have provided useful feedback and suggestions related to different aspects of this work, including Ben Caplan, Peter Culicover, Gregory Kierstead, Marie-Catherine de Marneffe, Dave Odden, Craige Roberts, Judith Tonhauser, the Ohio State Synners, and the audience at BLS 39. Special thanks go to Ben Caplan, Gregory Kierstead, and Judith Tonhauser, for comments on an earlier draft of this paper. I would also like to thank the Ohio State University Department of Linguistics and College of Arts and Sciences for financially supporting my fieldwork on Mushunguli. The greatest debt of gratitude is owed to my Mushunguli consultant. All errors are my own. 


\section{Jefferson Barlew}

for a detailed analysis of non-projective spatial expressions). Projective spatial expressions, on the other hand, relate the location of the Figure to that of the Ground in terms of a direction, as illustrated by behind in (1).

On one prominent reading, (1) asserts that the ball is located in a region in space in a particular direction from the car, specifically, near its back, the part where the tail lights and exhaust pipe are. The phrase behind the car is analyzed as denoting that region (or a set of such regions depending on the formal system; Zwarts and Winter 2000, Kracht 2008). Part of the meaning of behind itself is thus a function from an entity to a region. The meaning obviously must involve a direction as well, since only regions in a particular direction from the Ground can be described using behind. Where does this direction come from?

At least since Levinson (1996), the standard answer is that the direction comes from a frame of reference. A frame of reference (FoR) is a coordinate system consisting of a set of directions related to each other in particular ways. Levinson (1996, 2003) develops a typology of FoRs consisting of absolute, relative, and intrinsic FoRs, to which Danziger (2010, 2011), Bohnemeyer and O'Meara (2010), and Bohnemeyer $(2011,2012)$ suggest additions. In Levinson's typology, FoRs are differentiated by the ways in which their directions are determined and by which types of inference they license. In this paper, I focus on the former and ignore the latter (for discussions inference patterns associated with FoRs, see Levinson 1996, 2003 and Danziger 2010, 2011).

The entity that determines the directions of an FoR is called the anchor. Although Levinson $(1996,2003)$ assumes that only some FoRs involve anchors, Bohnemeyer (2012) argues convincingly that all FoRs require them. As an example of how anchors define FoRs, consider the intrinsic FoR, in which the Ground is the anchor and directions are determined based on its intrinsic features. Since the directions comprising intrinsic FoRs are up, down, front, back, left, and right, in order to define an intrinsic FoR, an entity must have features that can be used to define these directions. An entity like the car in (1) has such features: its canonical direction of motion, the way human users are positioned when using it, its canonical orientation with respect to gravity, etc. (see Fillmore 1975, Herskovits 1986, and Levinson 2003 for discussions direction determination). Thus, in the interpretation of (1) described above, the car is the Ground and the anchor, and the direction involved in the meaning of behind is part of an intrinsic FoR.

There is another reading of (1) which involves a different FoR, the relative FoR. On this reading, the region denoted by behind the car may not be at the car's intrinsic back. Instead, the region is near the side of the car that is farthest from the point of view of some observer, typically the speaker (consider example 1 with the continuation so I can't see it). On this reading, the coordinate system is defined with respect to the location of an observer looking at the car rather than features of the car itself. For example, the direction front is defined as the direction from the car toward the observer, back is defined as the direction away from the observer, etc. The two readings illustrate the difference between the intrinsic FoR, with the 
Ground as its anchor, and the relative FoR, with an observer as its anchor.

In formal analyses that take FoRs into account, the meanings of projective spatial expressions are assumed to include a function from an anchor to a particular direction in a particular FoR and then another function from that direction and an entity to a region (Kracht 2008, Bohnemeyer 2012). ${ }^{1}$ Spatial expressions are thus assumed to take anchors, not directions, as implicit arguments. Such accounts generate a new question: where does the anchor come from?

The only answer in the literature is Bohnemeyer's (2012) proposal that projective spatial expressions are indexical in the sense of Kaplan (1989), with the anchor as a parameter of the context of utterance. In this paper I argue instead that projective spatial expressions are anaphoric. I demonstrate that an anaphoric account makes better empirical predictions for projective spatial expressions in English. I then show that the anaphoric approach can be extended to account for a different type of spatial anchoring found in the meanings of non-projective spatial expressions in the Bantu language Mushunguli (Somalia).

\section{Bohnemeyer's (2012) indexical analysis of spatial expressions}

The difference between the two readings of (1) shows that the identity of the anchor and the type of FoR depend on the context rather than on the utterance itself. (2) also illustrates this point and demonstrates another motivation for an indexical analysis. Two contexts for (2) are depicted in Figure (3). (2) is felicitous and true in both contexts, but only on the assumption that the anchor differs from context to context.

(2) The ball is behind the bush.

(3) The speaker, addressee, Figure, and Ground in (2)

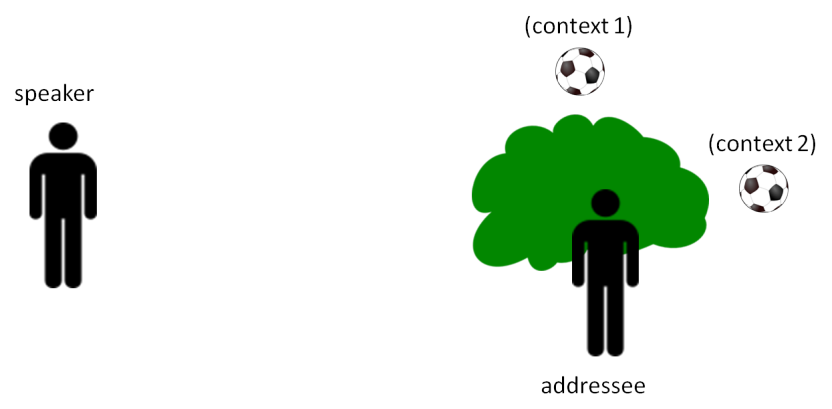

In context 1 , the addressee is searching for her lost ball. The speaker, who sees the ball, utters (2) in order to help the addressee locate it, using a relative FoR with the addressee herself as its anchor. In context 2, the speaker is attempting to shoot the ball with a BB-gun, and (2) might be followed by Please move it so that I can

\footnotetext{
${ }^{1}$ In Kracht's (2008) account, the first function differs from expression to expression and takes an unspecified set of contextual factors as its argument(s). If the anchor is assumed to be the source of these contextual factors, the account is similar to Bohnemeyer's.
} 


\section{Jefferson Barlew}

see it better. In context 2 , the speaker is the anchor. The context dependence of the anchor and the fact that in the relative FoR the anchor is often a discourse participant (Bohnemeyer 2012) are motivations for arguing that anchors are parameters of the context, and projective spatial expressions are indexical.

\subsection{Details of the indexical analysis}

In the Kaplanian (1989) account that Bohnemeyer (2012) proposes, the meanings of projective spatial expressions are complex. Part of the meaning of each such expressions is a "character," or a function from contexts of utterance to contents. A context of utterance is modeled as a tuple including parameters for the speaker, the utterance time, and other elements. To this tuple, Bohnemeyer proposes to add an anchor parameter. The character of a projective spatial expression is a function that returns the value of the anchor parameter of the context, just as the character of the indexical $I$ is a function that returns the value of the speaker parameter. It is this character that makes projective spatial expressions indexical.

Bohnemeyer formalizes the character of projective spatial expressions by introducing a constant, anchor, the denotation of which is determined by the interpretation function. In every context, the value of anchor is the anchor parameter of that context, $c_{a}$. Across FoRs, different expressions place different restrictions on the anchors they select, and thus on the contexts in which they are acceptable. For example, expressions involving directions from a relative FoR are said to require the anchor to be a (real or hypothetical) observer Levinson (2003: 47).

Another part of the meaning of a projective spatial expression is an "axis function," which takes the constant anchor as its argument and returns an ordered pair consisting of "the selected anchor and [a particular] axis projected onto the origin of the reference frame" (Bohnemeyer 2012: 25). The final part of the meaning of a projective spatial expression relates the location of the Ground to a region in space in which the Figure is located, as analyzed by Zwarts and Winter (2000). As an example of this analysis, the lexical entry for above is presented in (4).

(4) above' $:=\lambda A \cdot \lambda v \cdot \operatorname{ext}(v, A) \wedge c($ up(anchor $), v)>0$

(Bohnemeyer 2012: 22; 25-26)

Given the space occupied by the Ground, $A$, (4) returns a set of vectors, $v$, that start at $A$ and end external to $A(\operatorname{ext}(v, A))$. These vectors have a component, that is parallel to the axis defined by the axis function up applied to the constant anchor. The component is selected using the function $c$ applied to the axis and the vector $v$ : $c($ up(anchor $), v)>0$. Requiring the vectors $v$ to have this component insures that each vector ends at a point that is some distance above the Ground. For Bohnemeyer, as for Zwarts and Winter (2000), a set of vectors corresponds to a region. Thus the output of (4) is a region, characterized as a set of vectors. The indexicality of the projective spatial expression is captured in the interpretation of the anchor constant. Bohnemeyer demonstrates that this system provides accurate 


\section{Anchored to what? An anaphoric approach to frames of reference}

truth conditions for utterances involving a variety of FoRs including Levinson's original three types and the new "head-anchored" FoR described in Bohnemeyer and O’Meara (2010) and Bohnemeyer (2012).

\subsection{Predictions of the indexical analysis}

Assuming that the anchor is interpreted as a parameter of a Kaplanian context makes certain predictions. Three are listed in (5).

a. The interpretation of the anchor must be determined by the context of utterance. $^{2}$

b. Anchors cannot be quantificationally bound.

c. In a single utterance, the anchors of all projective spatial expressions must have the same interpretation.

(5a) is inherent in the definition of indexicality. (5b) is the case because parameters of the context cannot vary as quantified elements vary, the way bound variables must. Additionally, like the intensional operators (modals and temporal expressions) Kaplan (1989: 502) discusses, quantifiers are functions on contents, not contexts. (5c) is due to Kaplan's (1989: 510) prohibition against "monsters," which is his name for operators that change contextual parameters during the course of the interpretation of a single utterance. According to Kaplan, such operators do not exist in natural language. ${ }^{3}$ Analyzing projective spatial expressions as indexicals makes the three predictions in (5). However, these predictions are incorrect, as the data in Section 2.3 demonstrate.

\footnotetext{
${ }^{2}$ This prediction is technically too strong. As pointed out to me by Ben Caplan (p.c.), Predelli (1998 and subsequent work) argues that the context of interpretation may differ from the context of utterance and may also be the source of the value of a contextual parameter. However, in the examples in this paper, the context of utterance and context of interpretation are identical, resulting in this simplified prediction.

3 Recently, researchers working on languages other than English have shown that Kaplan's prohibition is too strong and have provided evidence that some operators can change parameters of the context (e.g. Schlenker 2003, Anand and Nevins 2004; Kierstead 2013; Deal to appear). Anand and Nevins (2004) and Deal (to appear) argue that some of these operators target only a single parameter of the context. The indexical account of the meanings of projective spatial expressions could be modified to include a context changing operator that targets only the anchor parameter. Bohnemeyer himself hints that such an operator may be necessary. He observes that within a single utterance different projective spatial expressions may have different anchors. He therefore proposes that each projective spatial expression is evaluated relative to a different context with a (potentially) different anchor. He does not, however, describe how the context shifts. If a context shifting operator that targets the anchor parameter exists, it behaves differently than other operators, which are often associated with propositional attitude predicates (see e.g. Kierstead 2013). Below, (9), which illustrates reference to multiple anchors in a single utterance, shows that no attitude predicate is necessary for a change in anchors to occur.
} 


\section{Jefferson Barlew}

\subsection{Testing the predictions of the indexical analysis}

Prediction (5a) is shown to be incorrect in (6). In both examples in (6), the anchor is not an element of the context of utterance.

(6) Context: Ernie and Jim are at school before class talking about their friends John, Marie, and Sarah, who are not present.

a. Ernie: John told me he went bowling yesterday. When he went to the rack to choose a ball, a pink ball caught his eye, but he ultimately chose a blue ball immediately to the right of it.

b. Jim: Sarah told me that Marie hid her softball and bat near a large tree. Eventually, Sarah spotted the softball in a clump of grass to the right of the tree. The bat, on the other hand, was behind the tree.

Both examples in (6) involve Grounds (ball and tree) without intrinsic axes in the horizontal plane. As a result, the felicitous use of the spatial expressions to the right of and behind involve relative FoRs. On the most natural reading of (6a) the anchor is John, and the second sentence means that the blue ball was to the right of the pink one from John's perspective as he stood in front of the rack. Similarly, on the most natural reading of (6b), the bat is behind the tree from Sarah's perspective at the time at which she spotted the ball, and thus Sarah is the anchor of behind in (6b). In both cases, the anchor is introduced and made salient by prior linguistic content, but is not an element of the context of utterance in Kaplan's narrow sense. (6b) demonstrates that the anchor need not be mentioned in the sentence containing the projective spatial expression, provided it is mentioned previously in the discourse.

Prediction (5b) is tested in (7), which shows that anchors can be quantificationally bound.

\section{(7) Context: Anna is describing place settings on round tables at a dinner.}

Anna: There is a fork to the left of every plate.

In (7), as in the examples in (6), the Ground (plate) has no intrinsic axes in the horizontal plane, preventing the use of an intrinsic FoR. In the examples in (6), a single perspective can be identified, and a single relative FoR constructed, relative to which each example is true. However, in (7), because the table is round, no such perspective can be identified. There is no single location on or around the table at which an observer can be positioned such that (7) will be true of all fork-plate pairs from that observer's perspective. However, depending on how the tables are actually set, someone in the room, or even someone elsewhere who knows about the dinner, can utter (7) truthfully. This is possible only if, for each fork-plate pair, a different perspective is assumed, presumably the perspective of a hypothetical observer sitting at that place at the table. Thus, the hypothetical observer's position varies as the plate varies under quantification, suggesting that the quantification 
over plates expressed by every binds the anchor of to the right of. With this quantificational binding of the anchor, the utterance is felicitous and, depending on the circumstances, true.

In addition to being quantificationally bound in single clause utterances like (7), anchor arguments can also be bound in donkey sentences. Classic donkey sentences involve a pronoun with an antecedent introduced by an NP that does not scope over the pronoun. In (8), the antecedent of the anchor of the projective spatial expression behind is a farmer, but a farmer does not scope over behind.

(8) Context: The staff psychologist of the retirement community for farmers who have beaten donkeys at least since Geach (1962) is a guest on a television talk show. She is talking to the host about what (hallucinating) farmers see when they look out the window.

Psychologist: Every tree that a farmer sees has a donkey behind it.

As in the examples above, the Ground in (8) has no intrinsic axes in the horizontal plane, requiring the relative FoR. The only interpretation available is the one in which a donkey is behind each tree from the perspective of the particular farmer looking at that tree. There is no salient farmer in the context of utterance, and there is no unique perspective, for example that of the speaker at utterance time, from which the direction involved in the meaning of behind can be defined for all relevant trees. Thus, the antecedent of the anchor is bound by the quantifier every. As the trees vary, the farmers vary, and so do the anchors.

Finally, (9) demonstrates that two different spatial expressions in a single utterance can involve to two different anchors, showing that prediction (5c) is incorrect.

(9) The ball is in front of the car [that is] behind the tree.

On a prominent reading of (9), the anchor of in front of is the car itself, yielding an intrinsic FoR, while the anchor of behind is a salient observer, yielding a relative FoR. This is counter to the prediction of the indexical approach, at least without an account of how the anchor parameter of the context shifts (see footnote 3 ). The data in this section demonstrate that indexical approach to frames of reference makes three incorrect predictions for the anchoring of projective spatial expressions.

\section{An anaphoric analysis of projective spatial expressions}

Instead of being interpreted indexically, I propose that anchors are interpreted anaphorically. This anaphoric analysis assumes a dynamic semantics based on the work of Kamp (1981) and Heim (1982, 1983) and its extension by Roberts (2002, 2003, 2005). In these frameworks, anaphoric expressions such as definite descriptions presuppose the existence of discourse referents. Discourse referents are informational entities present in the discourse context. As described by Roberts (2002: 16-17) the idea of discourse context in these theories is quite different from Kaplan's context of utterance, the tuple of parameters described above. The discourse 


\section{Jefferson Barlew}

context consists of all the information that the interlocutors share, or at least purport to share. The context thus includes, for example, a set of propositions to which the interlocutors are committed and a set of discourse referents (Roberts 2012).

The presence of a perceptible entity in the context of utterance can make a discourse referent corresponding to that entity available (Roberts 2003). As a result, anaphoric expressions can have antecedents that are introduced into the context simply by being perceptible to the interlocutors. Thus, anaphoric expressions, like indexicals, can be used to refer to an individual in the context of utterance. However, unlike contextual parameters, discourse referents can also be introduced linguistically and quantificationally bound (Heim 1982, Partee 1984, 1989, Condoravdi and Gawron 1996, Roberts 2002, 2003, 2005). In addition, unlike indexicals, which select the same contextual parameters across all uses within a single utterance, anaphoric expressions can have their presuppositions satisfied by different discourse referents across multiple uses in a single utterance.

While pronouns and definite NPs are the prototypical examples of anaphoric expressions, a wide range of expressions have been shown to involve implicit arguments that are interpreted anaphorically. Partee (1984) demonstrates that the meanings of many temporal expressions, including tense, involve implicit arguments that have temporal discourse referents as antecedents. Mitchell (1986), Partee (1989), and Condoravdi and Gawron (1996) show that a wide range of open class expressions, including the locational adjective local, have complex meanings with anaphoric components. In (10), local is used to exemplify the range of antecedents available for anaphoric expressions. (10a-10c) are from Condoravdi and Gawron's (1996: 5) example (8), itself based on examples by Partee.

(10) a. A local bar is selling cheap beer.

b. A reporter from the Times got seriously drunk. A local bar was selling cheap beer.

c. Every sports fan watched the Superbowl in a local bar.

d. Cindy, who lives in Cleveland, watched the Buckeye game at a local bar, whereas Bill, in Cincinnati watched it at a local coffee shop.

In the examples in (10), the meaning of local encodes that the location of the entity denoted by its argument is proximal to some reference location, typically the location of some other entity. The meaning of local involves an anaphorically interpreted implicit argument for that reference location. In the framework assumed here, that means that the meaning of local presupposes the existence of discourse referent for the reference location.

Without any prior context, (10a) is most naturally interpreted as encoding that the bar is proximal to the location of the speaker or the speaker's home base (see Fillmore 1975 for a discussion of location at utterance time compared to home base). On that reading, the antecedent of the implicit argument of local is a discourse referent corresponding the location of the speaker, an element of the context 


\section{Anchored to what? An anaphoric approach to frames of reference}

of utterance. On one reading of (10b), the bar is proximal to the reporter's location or home base. On this reading, the reporter's location is the antecedent. The antecedent is entailed to exist due to the existence of the discourse referent introduced linguistically by the indefinite NP a reporter. (10c) has a reading in which each fan watched the Superbowl in a bar that is proximal to her own location. Since fans are quantified over, the antecedent of local is quantificationally bound. Finally, on one reading of (10d), the implicit argument of the first occurrence of local is interpreted as Cindy's location, while that of the second is interpreted as Bill's location. Thus, different discourse referents satisfy the anaphoric presuppositions of different occurrences of local.

The similarity of the examples in Sections 1 and 2 to those in (10) motivates an anaphoric analysis of spatial expressions. On this analysis, the meanings of projective spatial expressions presuppose the existence of a discourse referent corresponding to an anchor. This analysis makes exactly the opposite predictions from those of the indexical approach given in (5), as shown in (11).

(11) a. The anchor can be introduced linguistically, and need not be determined by the context of utterance.

b. Anchors can be quantificationally bound.

c. In a single utterance, the anchors of different projective spatial expressions may have different interpretations.

These predictions are confirmed by the examples involving projective spatial expressions above. (6) confirms (11a); (7) and (8) confirm (11b); and (9) confirms (11c). The availability of anchors in the context of utterance, predicted by both accounts, is confirmed by (1) and (2).

To formalize this approach, I assume the dynamic semantics described in Roberts (2003). In that system, discourse referents are modeled as a set of numerical indices, Dom, with each index corresponding to a discourse referent. Dom is a subset of the set of natural numbers, N. There is also a set $\mathrm{G}$ of assignment functions, $g$, which are functions from $\mathrm{N}$ to the set of individuals. Applying an assignment function $g$ to a discourse referent $i$ returns an individual that verifies all of the information the interlocutors share about discourse referent $i$.

I analyze projective spatial expressions as presupposing the existence of a discourse referent, the interpretation of which can serve as the anchor. It is beyond the scope of this paper to determine what selectional requirements apply to anchors of particular projective spatial expressions. For example, presumably, the anchor of west is required to have different properties than the anchor of behind. As mentioned in Section 5, determining the range of such properties is the next step toward a fully developed anaphoric analysis of projective spatial expressions.

To avoid confusion and to highlight the key difference discussed here-the distinction between indexical and anaphoric interpretations of anchors-I follow Bohnemeyer in adopting Zwarts and Winter's (2000) vector space semantics, and 


\section{Jefferson Barlew}

retain Bohnemeyer's axis functions. The lexical entry for above under the anaphoric analysis is given in (12).

(12) a. The use of above is felicitous only if there exists some $i \in$ Dom the interpretation of which can serve as an anchor, and

b. above' $:=\lambda A \cdot \lambda v \cdot \operatorname{ext}(v, A) \wedge c(u p(g(i)), v)>0$

Other than the presupposition discussed above, the only difference between (12) and the lexical entry in (4) is that in (12) the axis function up applies to the interpretation of the $\mathrm{i}^{\text {th }}$ discourse referent rather than to the anchor constant. However, this change results in the prediction that examples (6)-(9) are acceptable, which is correct. This anaphoric analysis of the meanings of projective spatial expressions in English thus makes better empirical predictions than an indexical approach.

\section{Anaphoric reference to anchors in non-projective spatial expressions in Mushunguli}

In English, only projective spatial expressions presuppose the existence of an anchor. However, in the Bantu language Mushunguli (Somalia), some non-projective spatial expressions, e.g. the equivalents of English near and at, also presuppose the existence of a type of anchor. ${ }^{4}$ In this section, I demonstrate that the interpretation of the anchors of non-projective spatial expressions in Mushunguli is best analyzed by extending the anaphoric approach developed for projective expressions in English developed above.

In Mushunguli, spatial expressions often involve the use of one or more locative morphemes (Barlew 2012; for locative morphemes in Bantu languages generally, see Ružička 1959, 1960, Ziervogel 1971, inter alia). Barlew (2012) demonstrates that the locative morpheme ha- encodes proximity between its argument, which is either the Ground or a part of the Ground, depending on the construction, and an anchor. Like the implicit argument of local, the anchor of $h a$-is a salient location. Obligatory proximity to the anchor is illustrated in (13), where the anchor is the location of the interlocutors. ${ }^{5}$

(13) Context: A banana and a book are sitting on top of a pedestal. The book is 1-2m from the interlocutors.

$$
\begin{aligned}
& \text { i-di-boko di-i ha-nkhanda ha-a-i-chi-tabu } \\
& \mathrm{AUG}_{5} \text { - } \mathrm{CL}_{5} \text {-banana AGR } 5 \text {-COP LOC-side LOC-ASSOC-AUG }-\mathrm{CL}_{7} \text {-book }
\end{aligned}
$$

\footnotetext{
${ }^{4}$ Mushunguli is a severely under-documented language spoken by about 23,000 people (Lewis 2009). Holman Tse p.c. observes that some native speakers find the name Mushunguli offensive, preferring the name Kizigua. However, since my consultant refers to the language as Mushunguli, I do the same. The data used in this study were collected between 2010 and 2012 during original field work in Columbus, Ohio.

${ }^{5}$ Mushunguli has tone, but tone has not been found to be significant for locative constructions and is not indicated in the examples here. Glosses: $a g r_{\#}$ : agreement morpheme of class \#; ASSOC: associative morpheme (similar to genitive); $\mathrm{AUG}_{\#}$ : augment morpheme of class \#; $\mathrm{CL}_{\#}$ : class morpheme of class \#; COP: copula; LOC: locative.
} 
'The banana is beside the book.'

(13) involves the locative phrase ha-nkhanda ha-a-i-chi-tabu 'beside the book' which includes two instances of the non-projective locative morpheme ha-, the meaning of which is similar to the meaning of English at but which also encodes proximity to an anchor (Barlew 2012). The first instance of ha- combines with the locative stem -nkhanda 'side' to yield the non-projective spatial relational term hankhanda 'beside.' The proximity entailment and anchor of ha-nkhanda 'beside' are discussed in more detail below.

The second ha-combines with a-i-chi-tabu 'of the book,' a phrase including an NP referring to the Ground plus the associative morpheme, which is similar to the Indo-European genitive. In (13), the second $h a$ - encodes proximity between the book and an anchor. Here, the anchor is the location of the interlocutors. (13) is acceptable in the context given, in which the book is $1-2 \mathrm{~m}$ from the interlocutors, but unacceptable in a minimally different context in which the book is $25-30 \mathrm{~m}$ from the interlocutors. A minimally different sentence without ha-nkhanda 'beside' displays the same pattern of acceptability across contexts.

(13) demonstrates that the anchor for Mushunguli ha-can be an element of the context of utterance: the location of the interlocutors. As mentioned above, such anchoring is predicted by both indexical and anaphoric accounts.

Anchoring to a previously mentioned location, predicted by an anaphoric account but not by an indexical account, is illustrated in (14). Here, the locative phrase $k u-a-u-m u-t i$ 'at the tree' denotes the location of the tree, which serves as the anchor for ha-a-i-di-hanshi 'at the paper.' ${ }^{\prime}$

(14) Context: A paper and a banana are affixed side by side to the trunk of a large tree. The interlocutors have gone far enough away that they cannot see the tree.

$$
\begin{aligned}
& \text { i-di-boko di-i } \quad \boldsymbol{k u} \text {-a-u-mu-ti ha-nkhanda }
\end{aligned}
$$

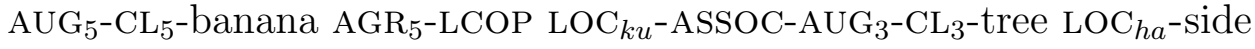

$$
\begin{aligned}
& \text { ha-a-i-di-hanshi } \\
& \mathrm{LOC}_{h a} \text {-ASSOC-AUG }{ }_{5}-\mathrm{CL}_{5} \text {-paper }
\end{aligned}
$$

'The banana is on the tree, beside the paper.'

In the context in (14), the locative phrase ha-nkhanda ha-a-i-di-hanshi 'beside the paper' is acceptable only when the location of the tree is mentioned in the discourse, as it is in (14). A minimally different utterance without $k u-a-u-m u-t i$ 'at the tree' is unacceptable in this context. This demonstrates that the anchor of $h a$ can be a discourse referent introduced into the discourse linguistically.

The anchor of $h a$ - can also be bound by quantification. This is demonstrated in (15) where the quantifier kakila 'always' binds the anchor argument.

${ }^{6}$ Unlike $h a$-, the locative morpheme $k u$ - does not involve a proximity requirement. See Barlew (2012) for details. 
Jefferson Barlew

(15) Context: Hasani and Hamadi are at a large sale where people are selling animals. They notice that the cows and the pigs are beside each other. Hasani, who has not been to one of these sales, asks Hamadi, who goes to many of them in various villages, if it is normal for the pigs to be beside the cows. Hamadi responds:

i-zi-nguluwe kakila zi-i ha-nkhandaha-a-ny-ngombe.

$\mathrm{AUG}_{10}-\mathrm{CL}_{10}$-pig always $\mathrm{AGR}_{10}$-COP LOC-side LOC-ASSOC-CL 10 -COW

'The pigs are always beside the cows.'

In (15), there is no single location such that all of the sets of pigs and cows quantified over is proximal to it. Nevertheless, the example, with the locative phrase ha-nkhanda ha-a-ny-ngombe 'beside the cows,' is acceptable. This acceptability indicates that the anchor varies with each instance of pigs being beside cows.

Thus far, I have ignored the anchor of the ha- in ha-nkhanda 'beside.' (16) provides evidence that, in phrases such as ha-nkhanda ha-a-i-chi-tabu 'beside the book' in (13), the ha- in ha-nkhanda 'beside' does not necessarily have the same anchor as the ha-ha-a-i-chi-tabu 'at the book.' The context in (16) is similar to that in (13), except that instead of a banana beside a book (16) involves a cloth beside a tree. The examples differ in that in (16) the only $h a$ - is in the word ha-nkhanda 'beside,' which allows the anchoring of that $h a$ - to be investigated independently of the anchoring of the following word.

(16) Context: An cloth is on the ground a few centimeters from a tree. The speaker and the addressee are standing 30-35m from the side of the tree opposite the cloth.

$$
\begin{array}{ll}
i-i & \boldsymbol{h a}-n k h a n d a k u-a-u-m-t i \\
\mathrm{AGR}_{9} \text {-COP } \mathrm{LOC}_{h a} \text {-side } \mathrm{LOC}_{k u} \text {-ASSOC-AUG }{ }_{3}-\mathrm{CL}_{3} \text {-tree }
\end{array}
$$

'It [the cloth] is beside the tree.'

(16) shows that, when the only ha- is in ha-nkhanda 'beside,' the location of the Ground may be distant from that of the interlocutors. Thus, (16) differs from (13), which has two instances of ha- (ha-nkhanda ha-a-i-chi-tabu 'beside the book'), and which is acceptable only if the Ground is proximal to the location of the interlocutors. Assuming a unified analysis of $h a-$, the $h a$ - in ha-nkhanda 'beside' in (16) must have an anchor. The anchor is not the location of the interlocutors but rather the only other salient location in the discourse, the location introduced linguistically by $k u-a-u-m-t i$ 'at the tree.' The meaning of ha-nkhanda 'beside' in (16) thus encodes that the side of the tree, denoted by -nkhanda 'side,' is proximal to the location of the tree.

If meanings compose in the same way across examples such as (13) and (16), the fact that the anchor of $h a$ - in (16) is the location denoted by $k u-a-u$ - $m u$ - $t i$ 'at the tree' suggests that the ha- in ha-nkhanda 'beside' in (13) is anchored to the location 
of the book, denoted by ha-a-i-chi-tabu 'at the book.' The ha- in ha-a-i-chi-tabu 'at the book,' on the other hand, is anchored to the location of the interlocutors, as shown above. Thus, taken together, (13) and (16) suggest that two instances of $h a$ - in a single utterance may have different anchors, just as two projective spatial expressions in English may have different anchors, and just as is predicted by an anaphoric account.

The examples in this section demonstrate that anchors for the Mushunguli nonprojective spatial expression $h a$ - display the same range of interpretations as anchors of projective spatial expressions in English do. An anchor can be a salient location in the context of utterance or a location referred to previously in the discourse, an anchor can be quantificationally bound, and a given utterance can involve more than one anchor. Thus, both the Mushunguli data and the English data match the predictions of an anaphoric account rather than those of an indexical account.

\section{Conclusions}

In this paper, I have argued that reference to anchors in spatial expressions is anaphoric. I have demonstrated that range of interpretations possible for the anchors of spatial expressions can be accounted for by assuming the anchor argument is interpreted anaphorically, rather than indexically. However, the details of the anaphoric analysis of spatial expressions have yet to be worked out. In particular, I have not given an account of the factors that make one discourse referent more likely than another to be the antecedent of a spatial expression. In contrast, in her anaphoric analysis of English definite NPs, Roberts (2003) delineates presuppositions associated with definites which, combined with a suitable theory of discourse structure such as that in Roberts (2012), can be used to predict which discourse referent will satisfy the presuppositions of a given definite NP, given a discourse context. Discovering the details of the anaphoric presuppositions encoded by particular spatial expressions constitutes the next step in developing the anaphoric account of their meanings.

\section{References}

Anand, Pranav and Nevins, Andrew, 2004. Shifty operators in changing contexts. In R. Young, ed., SALT XIV, 20-37.

Barlew, Jefferson, 2012. The semantics and pragmatics of Mushinguli locatives. Ms. The Ohio State University.

Bohnemeyer, Jürgen, 2011. Spatial frames of reference in Yucatec: Referential promiscuity and task-specificity. Language Sciences 33:892-914.

Bohnemeyer, Jürgen, 2012. A vector space semantics for reference frames in $\mathrm{Yu}$ catec. In E. Bogal-Allbritten, ed., Proceedings of the Sixth Meeting on the Semantics of Under-represented Languages in the Americas and SULA-Bar, 


\section{Jefferson Barlew}

15-35. Amherst: Graduate Linguistics Student Association, University of Massachusetts.

Bohnemeyer, Jürgen and O'Meara, Carolyn, 2010. Vectors and frames of reference: Evidence from Seri Yucatec. talk at STALDAC 2010.

Condoravdi, Cleo and Gawron, Jean Mark, 1996. The Context Dependency of Implicit Arguments. In Quantifiers, Deduction, and Context. Palo Alto: CSLI Publications.

Danziger, Eve, 2010. Deixis, gesture, and cognition in spatial frame of reference typology. Studies in language 34(1):167-185.

Danziger, Eve, 2011. Distinguishing three-dimensional forms from their mirrorimages: Whorfian results from users of intrinsic frames of linguistic reference. Language Sciences .

Deal, Amy Rose, to appear. Nez Perce embedded indexicals. In Proceedings of SULA 7: Semantics of Under-Represented Languages in the Americas. Amherst, MA: University of Massachusetts.

Fillmore, Charles J., 1975. Santa Cruz lectures on Deixis. Bloomington, Indiana: Indiana University Linguistics Club.

Geach, Peter, 1962. Reference and Generality: An Examination of Some Medieval and Modern Theories. Ithaca, NY: Cornell UP.

Heim, Irene, 1982. The Semantics of Definite and Indefinite Noun Phrases. Ph.D. thesis, University of Massachusett, Amherst.

Heim, Irene, 1983. File change semantics and the familiarity theory of definiteness. In Meaning, Use, and the Interpretation of Language, 164-190. Berlin: Walter de Gruyter.

Herskovits, Annette, 1986. Language and Spatial Cognition: An Interdisciplinary Study of the Prepositions in English. Cambridge: Cambridge University Press.

Kamp, Hans, 1981. A Theory of Truth and Semantic Representation. In J. Groenendijk, T. Janssen, and M. Stokhof, eds., Formal Methods in the Study of Language, 277-322. Amsterdam: Mathematische Centrum.

Kaplan, David, 1989. Demonstratives. In J. Almog, J. Perry, and H. Wettstein, eds., Themes from Kaplan. New York: Oxford University Press.

Kierstead, Gregory, 2013. The shifted indexical behavior of Tagalog sana 'wish'. Talk presented at the 49th Annual Meeting of the Chicago Linguistic Society.

Kracht, Marcus, 2002. On the Semantics of Locatives. Linguistics and Philosophy 25:157-232.

Kracht, Marcus, 2008. The fine structure of spatial expressions. In A. Asbury, J. Dotlacil, B. Gehrke, and R. Nouwen, eds., Syntax and Semantics of Spatial $P$, 35-62. Amsterdam/Philadelphia: Benjamins.

Levinson, Stephen C., 1996. Frames of reference and Molyneux's question: Crosslinguistic evidence. In P. Bloom, M. A. Peterson, L. Nadel, and M. F. Garrett, eds., Language and Space. Cambridge, MA: MIT Press.

Levinson, Stephen C., 2003. Space in Language and Cognition: Explorations in Cognitive Diversity. Cambridge: Cambridge University Press. 
Anchored to what? An anaphoric approach to frames of reference

Lewis, Paul M., 2009. Ethnologue: Languages of the World. Dallas, TX: SIL International, 16th edition.

Mitchell, Jonathan Edward, 1986. The Formal Semantics of Point of View. Ph.D. thesis, University of Massachusetts.

Partee, Barbara H., 1984. Nominal and temporal anaphora. Linguistics and Philosophy 7:243-286.

Partee, Barbara H., 1989. Binding Implicit Variables in Quantified Contexts. In Papers of the Chicago Linguistic Society, volume 25, 342-365.

Predelli, Stefano, 1998. I am not here now. Analysis 58(2):107-115.

Roberts, Craige, 2002. Demonstratives as Definites. In Information Sharing: Reference and Presupposition in Language Generation and Interpretation. Stanford: CSLI.

Roberts, Craige, 2003. Uniqueness in Definite Noun Phrases. Linguistics and Philosophy 26:287-350.

Roberts, Craige, 2005. Pronouns as Definites. In M. Reimer and A. Bezuidenhout, eds., Descriptions and Beyond, 503-543. Cambridge: Oxford UP.

Roberts, Craige, 2012. Information structure in discourse: Towards an integrated formal theory of pragmatics. Semantics and Pragmatics 5(6):1-69.

Ružička, Karel F., 1959. Locative formations in Bantu languages. Part I: Morphology of the locative. Archiv Orientalni 27:208-250, 604-669.

Ružička, Karel F., 1960. Locative formations in Bantu languages. Part II: Syntax of the locative. Archiv Orientalni 28:208-250, 604-669.

Schlenker, Phillipe, 2003. A Plea for monsters. Linguistics and philosophy 26:29120.

Talmy, Leonard, 1985. Lexicalization patterns: Semantic structure in lexical forms. In T. Shopen, ed., Language Typology and Syntactic Description, volume 3. Cambridge: Cambridge University Press.

Ziervogel, D, 1971. The Bantu locative. African Studies 30:371-384.

Zwarts, Joost and Winter, Yoad, 2000. Vector space semantics: A model-theoretic analysis of locative prepositions. Journal of Logic, Language and Information 9:161-211. 